\title{
Constraining cosmological parameters through Sunyaev-Zel'dovich surveys
}

\author{
SUBHABRATA MAJUMDAR ${ }^{1,2}$ and RAVI SUBRAHMANYAN ${ }^{3,4}$ \\ ${ }^{1}$ Joint Astrophysics Programme, Department of Physics, Indian Institute of Science, \\ Bangalore 560 012, India \\ ${ }^{2}$ Indian Institute of Astrophysics, Koramangala, Bangalore 560 034, India \\ ${ }^{3}$ Australia Telescope National Facility, CSIRO, Locked bag 194, Narrabri, NSW 2390, Australia \\ ${ }^{4}$ Raman Research Institute, Sadashivanagar, Bangalore 560 080, India
}

\begin{abstract}
For cold dark matter models, images of temperature fluctuations in the cosmic microwave background (CMB), due to Sunyaev Zel'dovich (SZ) effect have been been simulated taking a cosmolgical distribution of clusters into account. All the models are normalised to the 4-year COBE data. The image statistics are compared with the ATCA limits on arcmin scale anisotropy. The comparison appears to favour low- $\Omega_{0}$ open universe models.
\end{abstract}

Keywords. Cosmic microwave background; clusters; cosmology theory; cosmology observations.

PACS Nos 98.65.Cw; 98.65.Dx; 98.70.Vc

\section{Introduction}

Anisotropies in the CMBR are generally divided broadly into two categories, i.e. primary and secondary anisotropies. The primary anisotropies are due to scattering at the last scattering surface. The secondary anisotropies arise due to scattering of the background photons as they travel from the last scattering surface to the present time. Among the various effects giving anisotropies on small angular scales, we focus on SZ effect. SZ effect [1] occurs when the hot intracluster gas inverse scatters the passing microwave photons, where the electrons in the gas transfer energy to the CMB photons passing through the cluster, and this in turn distorts the CMB spectrum. This effect is observable as a change in sky brightness towards the cluster with respect to the mean background intensity. The magnitude of the distortion is given by the Compton y-parameter.

$$
y=\int \mathrm{d} l \frac{K\left(T_{e}-T_{\gamma}\right)}{m_{e} c^{2}} n_{e} \sigma_{T} .
$$

Here $T_{e}, n_{e}$ and $m_{e}$ refer to the electron temperature, number density and mass respectively in the cluster and $T_{\gamma}$ is the microwave temperature at redshift $z . \sigma_{T}$ is the Thomson scattering coefficient. Note that $y$ is dimensionless. 
In SZ effect, the surface brightness of the fluctuation has the major advantage of being independent of distance. It has another advantage due to the fact that for the integrated SZ effect due to an object, its flux density is proportional to the total hot gas mass times the particle weighted temperature. Therefore, the distortion is independent of the gas spatial distribution for unresolved clusters (for details see Bartlett [2] and the references within). In this work we use a SZ blank sky survey to put limits on cosmological parameters. Throughout the work vacuum energy parameter $\Lambda=0$, and the Hubble constant $H=100 \mathrm{~h} \mathrm{~km} / \mathrm{s} / \mathrm{Mpc}$.

\section{Computing the microwave background statistics from $\mathrm{SZ}$ effect}

\subsection{Cluster distribution and COBE normalisation}

For our simulation we have to relate the number density of collapsed virialised objects to the initial density contrast. Under the assumption of initial Gaussian density perturbations, the mass and redshift distribution of clusters is given by the Press-Schechter mass function [3]

$$
n(M, z) \mathrm{d} M=\sqrt{\frac{2}{\pi}} \frac{\rho_{\text {mean }}}{M} \nu_{c}\left|\frac{\mathrm{d} \ln \sigma(M)}{\mathrm{dln} M}\right| \mathrm{e}^{-\nu^{2} / 2} \frac{\mathrm{d} M}{M},
$$

where, written explicitly,

$$
\nu_{c}(M, z)=\frac{\delta_{c}(z, \Omega, \lambda)}{\sigma(M)} \frac{D_{g}(0, \Omega, \lambda)}{D_{g}(z, \Omega, \lambda)} .
$$

$D_{g}$ is the growth function and $\delta_{c}=1.68$ [4].

$$
\sigma^{2}(R, z)=\int_{0}^{\infty} \frac{\mathrm{d} k}{k} \frac{k^{3}}{2 \pi^{2}} P(k)\left|\tilde{W}_{R}(k)\right|^{2},
$$

where $\tilde{W}_{R}(k)$ is the Fourier transform of a real space window function and $M_{R}=$ $(4 \pi / 3) \rho_{c} \Omega_{0} R^{3}$ is the mass inside the window. Note that due to the exponential term in the mass function, the cosmic abundance of high mass objects are extremely sensitive to the amplitude and slope of power spectrum and the growth function.

For our work we have taken the following transfer spectrum [5]

$$
T_{\mathrm{CDM}}(q)=\frac{\ln (1+2.34 q)}{2.34 q} \times\left[1+3.89 q+16.1 q^{2}+5.46 q^{3}+6.71 q^{4}\right]^{-1 / 4}
$$

with $q=k / h \Gamma$ and $\Gamma=\Omega_{0} h \exp \left(-\Omega_{B}-\Omega_{B} / \Omega_{0}\right)$. The power spectrum is constructed from the transfer function in the usual way

$$
\frac{k^{3}}{2 \pi^{2}} P(k)=\left(\frac{c k}{H_{0}}\right)^{3+n} \delta_{H}^{2} T^{2}(k) D_{g}^{2}(z) / D_{g}^{2}(0),
$$

where $n$ is the index of primordial power spectrum and $\delta_{H}$ is the amplitude of perturbation on the horizon scale at $z=0$. COBE normalisation is done following Bunn and White [6]. 


\subsection{Generating the images}

We have simulated square patches of sky of side $42^{\prime} 40^{\prime \prime}$ which is subdivided into 256 pixels of $10^{\prime \prime}$ and at each redshift slice the clusters are randomly Poisson distributed. The random distribution of masses generates random images of the sky as should be seen by a telescope in a blank sky survey, an ensemble of which is needed for this study. For resolved clusters, the density profile of the cluster is given by the isothermal $\beta$ model.

Upon the assumption self similar collapse, the core radius $r_{c}$ and cluster temperature $T$ has the following scaling law

$$
\begin{aligned}
& r_{c}\left(\Omega_{0}, M, z\right)=\frac{1.3 \mathrm{~h}^{-1} \mathrm{Mpc}}{p} \frac{1}{(1+z)}\left[\frac{M}{10^{15} \mathrm{~h}^{-1} \mathrm{M}_{\odot}} \frac{\Delta\left(\Omega_{0}=1, z=0\right)}{\Omega_{0} \Delta\left(\Omega_{0}, z\right)}\right]^{1 / 3}, \\
& T=6.7 \times 10^{7}(1+z)\left[\frac{M}{10^{15} \mathrm{~h}^{-1} \mathrm{M}_{\odot}}\right]^{2 / 3} \times\left[\frac{\Omega_{0} \Delta\left(\Omega_{0}, z\right)}{\Delta\left(\Omega_{0}=1, z=0\right)}\right]^{1 / 3} \mathrm{~K} .
\end{aligned}
$$

Here the nonlinear density contrast at virialisation is $\Delta=\tilde{\rho} / \rho$ where $\tilde{\rho}$ is the perturbation density and $\rho$ is the background density. (see, Colafrancesco et al [7]). For the no-evolution case, the gas mass fraction is assumed to be roughly 0.1 of the total mass. Knowing the impact parameter of the photons travelling through the cluster (w.r.t centre), the $y$ parameter at each pixel can be calculated. The SZ decrement at each pixel is simply given by $\Delta T / T=-2 y$. Once the cumulative decrement at each pixel is obtained for a distribution of clusters, the variance is calculated, both before and after convolution with the ATCA beam pattern at $8.7 \mathrm{GHz}$. Typical simulated images are shown in figure 1 . The simulation results can then be compared with the observational limits on arcmin scale CMB anisotropy set by the ATCA experiment $[8,9]$.

\section{Results and conclusion}

Once the simulations are complete, and the statistics are calculated after convolving the simulated sky with the ATCA beam, comparison with present observations can be made. There appears a general decrease in the variance of the temperature anisotropy due to the SZ decrement as we go down from higher to lower effective shape factor (or lower $\Omega_{0}$ ). The expected variance obtained for SCDM model is far more than the limit set by ATCA observations which is about $20 \mu \mathrm{Jy}$ per beam. The variance drops down as one goes from a flat universe to an open universe, and falls below the ATCA limit around $\Omega_{0}=0.4$.

A comparison between the SCDM model and an open $\operatorname{CDM}\left(\Omega_{0}=0.4\right)$ is illustrated in the table. Thus the present data seems to disfavour the standard CDM scenario. Note that $\mathrm{SZ}$ effect is generically non-gaussian and the negative-skewed-nature of SZ effect may be used to distinguish it from other 'contaminents', 'foregrounds' and instrument noise. 


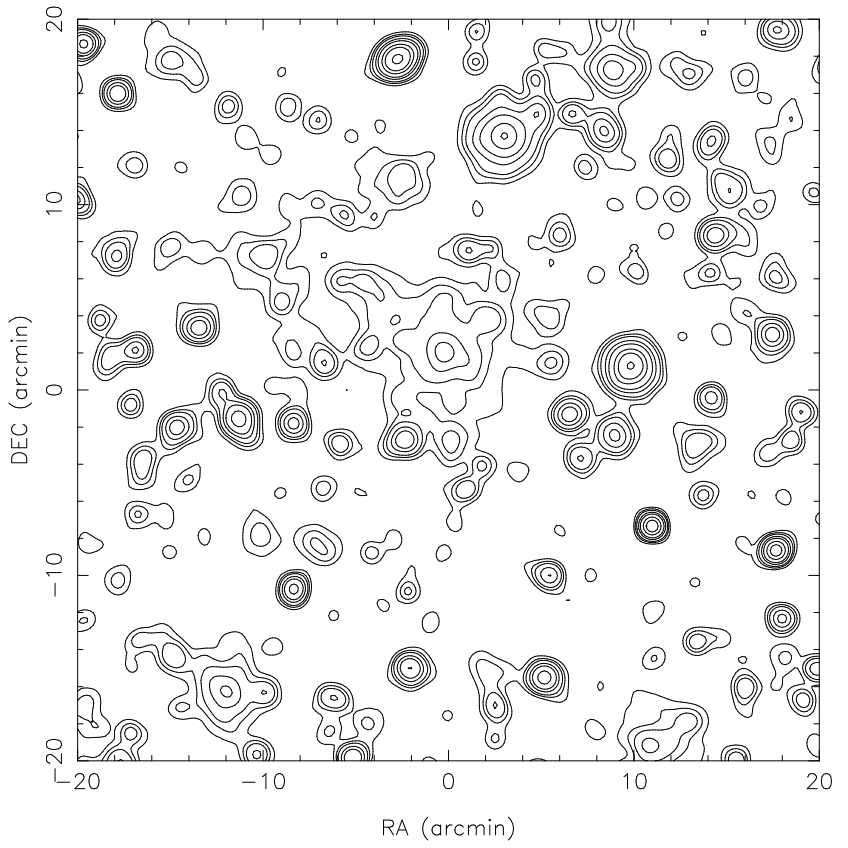

(a)

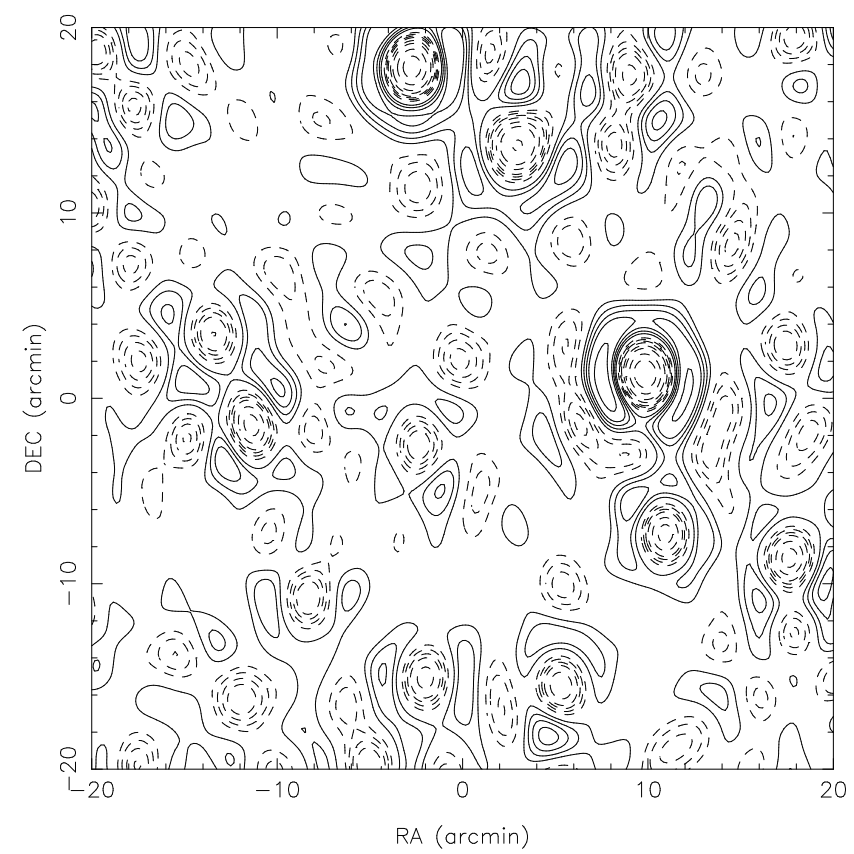

(b)

Figure 1a, b. A typical image: Figure (a) shows an 1 arcmin gaussian convolved image with contours at $-100 \mu \mathrm{K} \times(2,3,4,6,8,12,16,24)$. Figure (b) shows an ATCA beam convolved image with contours at $50 \mu \mathrm{Jy} / \mathrm{beam} \times(-16,-12,-8,-4,-2,-1,1,2$, $3,4,6)$. 
Table 1. Comparison of variance after convolution.

\begin{tabular}{lcccl}
\hline Model & $\Omega_{0}$ & $h$ & $\sigma_{8}$ & Variance $(\mu \mathrm{Jy})$ \\
\hline sCDM & 1.0 & 0.65 & 1.57 & 58.2 \\
oCDM & 0.4 & 0.65 & 0.65 & 3.5 \\
\hline
\end{tabular}

\section{Acknowledgements}

SM wants to thank the organisers of the workshop for a stimulating environment. He also wishes to thank Dr Pijushpani Bhattacharjee for making it possible to attend the workshop.

\section{References}

[1] R A Sunyaev, and Ya B Zel'dovich, Comm. Astrophys. Space Phys. 4, 173 (1972)

[2] J G Bartlett, From quantum fluctuations to cosmological structures, School held in Casablanca, edited by D ValsGabaud, M A Hendry, P Molaro and K Chamcham, A S P Conference Series, vol. 126, 1997

[3] W H Press, and P Schecter, Astrophys. J. 187, 225 (1974)

[4] P J E Peebles, Large scale structure of the universe (Princeton University Press, 1980)

[5] J M Bardeen, J R Bond, N Kaiser and A S Szalay, Astrophys. J. 304, 15 (1986)

[6] E F Bunn and D White, Astrophys. J. 480, 6 (1997)

[7] S Colafrancesco, P Mazzotta, Y Rephaeli and N Vittorio, Astrophys. J. 479, 1 (1997)

[8] R Subrahmanyan, R D Ekers, M Sinclair and J Silk, Mon. Not. R. Astron. Soc. 263, 416 (1993)

[9] R Subrahmanyan, M J Kesteven, R D Ekers, M Sinclair and J Silk, stro-ph/9 805245

[10] N Kaiser, Mon. Not. R. Astron. Soc. 222, 323 (1986) 